\title{
Application of the Generalized Simplest Equation Method to the Burgers Equation
}

\author{
Yuexing Bai, Sudao Bilige*, Xiaoqing Gao, Jianqing Lü \\ College of Sciences, Inner Mongolia University of Technology, Hohhot, China \\ Email: *inmathematica@126.com
}

How to cite this paper: Bai, Y.X., Bilige, S., Gao, X.Q. and Lü, J.Q. (2017) Application of the Generalized Simplest Equation Method to the Burgers Equation. Journal of Applied Mathematics and Physics, 5, 101109.

http://dx.doi.org/10.4236/jamp.2017.51011

Received: October 1, 2016

Accepted: January 22, 2017

Published: January 25, 2017

Copyright $\odot 2017$ by authors and Scientific Research Publishing Inc. This work is licensed under the Creative Commons Attribution International License (CC BY 4.0).

http://creativecommons.org/licenses/by/4.0/

\begin{abstract}
We successfully constructed wide classes of exact solutions for the Burgers equation by using the generalized simplest equation method. This method yielded a Bäcklund transformation between the Burgers equation and a related constraint equation. By dealing with the constraint equation, we obtained the traveling wave solutions and non-traveling wave solutions of the Burgers equation.
\end{abstract}

\section{Keywords}

The Generalized Simplest Equation Method, Exact Solution, the Burgers Equation

\section{Introduction}

The investigation of exact solutions to nonlinear evolution equations (NLEEs) plays an important role in the study of nonlinear physical phenomena. In the past several decades, many methods for obtaining exact solutions of NLEEs have been presented. Such as the inverse scattering method [1], the Hirota bilinear method [2], the Bäcklund transformation method [3], the homogeneous balance method [4], the Tanh-function method [5], the F-expansion method [6], the Exp-function method [7], the (G'/G)-expansion method [8] [9], the simplest equation method [10] [11] and other algebraic methods [12] [13] [14]. These methods are very effective for obtaining exact solutions of NLEEs, especially, for the traveling wave solutions.

Particularly, Ma et al. introduced the idea of transforming complicated partial differential equations (PDEs) into simple PDEs in the literature [15] and all such expansions, such as the extended tanh-function method and the simplest equation method, are concluded into the transformed rational function method. This method provides a more systematical and convenient handling of the solution 
process of NLEEs, unifying the tanh-function type methods, the homogeneous balance method, the exp-function method, the mapping method and the F-expansion type methods [16]. In addition, by using the multiple exp-function method, multiple wave solutions can be generated definitely based on Fourier theory [17]. Other interesting solutions methods include many new bilinear techniques [18] and the invariant subspace method [19]. But up to now, a unified method that can be used to deal with all types of NLEEs has not been discovered. Hence, developing new method and finding more general exact solutions of NLEEs have drawn a lot of interests of a diverse group of scientists.

For obtaining more general form exact solutions of NLEEs, recently we introduced a new direct method called the generalized simplest equation method to look for exact solutions of NLEEs [20]. In the method, we chose a solution expression with a variable coefficient and a variable coefficient ordinary differential auxiliary equation. This method can yield a Bäcklund transformation between NLEEs and a related constraint equation. By dealing with the constraint equation, we can derive an infinite number of exact solutions for NLEEs. These solutions include the traveling wave solutions, non-traveling wave solutions, multi-soliton solutions, rational solutions and other types of solutions. This method has its own advantages [20] which are different from other methods, and it can derive new exact solutions of NLEEs which are not obtained by using other methods [5]-[14]. In this paper, we will use this method to construct more exact solutions of the Burgers equation.

\section{The Exact Solutions of the Burgers Equation}

Let us consider the Burgers equation

$$
u_{t}+u u_{x}-c u_{x x}=0 \text {, }
$$

where $c$ is constant.

\subsection{The General Procedure of the Method}

First we show the general procedure of the generalized simplest equation method. The method is consisted of the following four steps.

Step 1. We introduce the solutions of Equation (1) that can be written as the following form

$$
u(x, t)=a_{0}(x, t)+a_{1}(t, x) \phi(\xi),
$$

where $a_{0}(t, x), a_{1}(t, x)$ will be determined later and the function $\phi=\phi(\xi)$ satisfies a first order ordinary differential Equation (ODE) with variable coefficients:

$$
\xi^{2}\left(\phi^{\prime}+\phi^{2}\right)+\delta \xi \phi+v=0
$$

where $\delta, v$ are constants.

Step 2. We substitute (2) along with Equation (3) into Equation (1), and collect all terms with the same order of $\phi$. As a result, the left-hand sides of Equation (1) are converted into another polynomials in $\phi$. Let each coefficient of 
powers of $\phi$ to be zero to get a set of over-determined PDEs with respect to $a_{0}(t, x), a_{1}(t, x)$ and $\xi(t, x)$.

Step 3. Using Mathematica to solve PDEs that have obtained in Step 2, we obtain the following solutions of $a_{0}(t, x), a_{1}(t, x)$ :

$$
a_{0}(t, x)=\left(c \delta \xi_{x}^{2}-c \xi \xi_{x x}-\xi \xi_{t}\right) / \xi \xi_{x}, a_{1}(t, x)=2 c \xi_{x},
$$

where the function $\xi=\xi(t, x)$ satisfies the constraint equation of $\xi$ as follow

$$
\begin{gathered}
c^{2} s\left(\xi_{x}^{2}-\xi \xi_{x x}\right) \xi_{x}^{4}-\xi^{3}\left[\xi_{x}^{2}\left(c^{2} \xi_{x x x x}+2 c \xi_{x x t}+\xi_{t t}\right)-2\left(2 c \xi_{x x}+\xi_{t}\right)\left(\xi_{x t}+c \xi_{x x x}\right) \xi_{x}\right. \\
\left.+\left(\xi_{t}^{2}+3 c^{2} \xi_{x x}^{2}+4 c \xi_{t} \xi_{x x}\right) \xi_{x x}\right]=0 .
\end{gathered}
$$

If consider the case of $\xi_{x} \neq 0$, we can get a more general form of solutions. Any solution of the constraint Equation (5) can be derived a set of coefficients (4) which result in three types of exact solutions (2) of Equation (1), as follows:

$$
u_{i}(t, x)=a_{0}(t, x)+a_{1}(t, x) \phi_{i}(\xi),
$$

where $\phi_{i}(\xi)$ are three types of general solutions of Equation (3) as follows:

$$
\begin{gathered}
\phi_{1}(\xi)=\varepsilon \cdot \frac{\left[(1-\delta) c_{1}-2 \eta c_{2}\right] \sin (\eta \ln |\xi|)+\left[2 \eta c_{1}+(1-\delta) c_{2}\right] \cos (\eta \ln |\xi|)}{2 \xi\left[c_{1} \sin (\eta \ln |\xi|)+c_{2} \cos (\eta \ln |\xi|)\right]}, i=1, s>1, \\
\phi_{2}(\xi)=\varepsilon \cdot \frac{c_{1}(2 \eta-\delta+1)|\xi|^{2 \eta}-c_{2}(2 \eta+\delta-1)}{2|\xi|\left(c_{1}|\xi|^{2 \eta}+c_{2}\right)}, \quad i=2, s<1, \\
\phi_{3}(\xi)=\varepsilon \cdot \frac{(1-\delta)\left(c_{1}+c_{2} \ln |\xi|\right)+2 c_{2}}{2 \xi\left(c_{1}+c_{2} \ln |\xi|\right)}, \quad i=3, s=1,
\end{gathered}
$$

where $c_{1}, c_{2}$ are constants and

$$
s=2 \delta-\delta^{2}+4 v, \quad \eta=|1-s|^{1 / 2} / 2,
$$

and if $\xi>0$, then $\varepsilon=1$; if $\xi<0$, then $\varepsilon=-1$.

The solution expression (6) has established a Bäcklund transformation between Equation (1) and the constraint Equation (5). By using the Bäcklund transformation, it is easy for us to obtain infinite number of exact solutions for the Burgers equation. Therefore, if we get the solution of the constraint Equation (5), the more general form exact solutions of Equation (1) will be obtained.

Step 4. In the following, we will solve the constraint Equation (5) and obtain the exact traveling wave and non-traveling wave solutions of Equation (1).

\subsection{The Exact Traveling Wave Solutions}

In this section, in order to find the exact traveling wave solutions of Equation (1), we define $\xi(t, x)=\xi(x-V t)$, then Equation (5) becomes the following form

$$
\begin{aligned}
& \xi(z)^{3}\left[4 \xi^{\prime}(z) \xi^{\prime \prime}(z) \xi^{(3)}(z)-\xi^{\prime}(z)^{2} \xi^{(4)}(z)-3 \xi^{\prime \prime}(z)^{3}\right] \\
& +S\left[\xi^{\prime}(z)^{2}-\xi(z) \xi^{\prime \prime}(z)\right] \xi^{\prime}(z)^{4}=0
\end{aligned}
$$

where $z=x-V t$. We have given twelve kinds of exact solutions for Equation 
(11) in literature [20]. We substitute these solutions $\xi=\xi(x-V t)$ into (7)-(9), (4) and assemble them in (6), we can get twelve kinds of exact traveling wave solutions of Equation (1). In the following, we only give three kinds of exact travelling wave solution of Equation (1).

Case 1: If $s>1$, we only give two kinds of exact travelling wave solutions as follows.

\section{Subcase 1.1:}

When $d_{2}^{2}+d_{3}^{2}-d_{1}^{2}>0, \quad A=\sqrt{d_{2}^{2}+d_{3}^{2}-d_{1}^{2}}, \quad A_{1}=d_{2}+\exp \left[d_{3}\left(z+d_{4}\right) \delta_{0}\right]$,

$$
\xi(z)=\left\{\begin{array}{r}
\varepsilon \exp \left[-\frac{2 \varepsilon}{\delta_{0}} \arctan \left(\frac{A \tan \left(\frac{A}{2} \delta_{0}\left(z+d_{4} \varepsilon\right)\right)+d_{3} \varepsilon}{d_{1}-d_{2}}\right)\right], \text { when } d_{1} \neq d_{2} \\
\varepsilon \exp \left[\frac{2}{\delta_{0}} \arccos \left(\frac{ \pm d_{3}}{\sqrt{d_{2}^{2}+d_{3}^{2}+\exp \left(d_{3} \delta_{0}\left(z+d_{4}\right)\right)\left(d_{2}+A_{1}\right)}}\right)\right], \\
\text { when } d_{1}=d_{2}
\end{array}\right.
$$

(I) If $d_{1} \neq d_{2}$, then we get $a_{0}(t, x), a_{1}(t, x)$ as follows by substituting $\xi(z)$ into (4).

$$
\begin{aligned}
a_{0}(t, x)= & \operatorname{sech} B_{5}^{2}\left[B_{3} V+2 A^{2} c \varepsilon B_{4}+B_{2} V \cosh \left(2 B_{5}\right)\right. \\
& \left.+c A B_{2} \delta_{0} \sinh \left(2 B_{5}\right)\right] / 2\left[\left(d_{1}-d_{2}\right)^{2}+A^{2} \tanh B_{5}^{2}\right] \\
a_{1}(t, x)= & -\frac{2 A^{2} c\left(d_{1}-d_{2}\right) \exp \left(-\frac{B_{1}}{\delta_{0}}\right) \operatorname{sech}\left(d_{3} \varepsilon+\frac{1}{2} \delta_{0} B_{0}\right)^{2}}{\left(d_{1}-d_{2}\right)^{2}+A^{2} \tanh B_{5}^{2}}
\end{aligned}
$$

where $B_{0}, B_{1}, B_{2}, B_{3}, B_{4}, B_{5}$ can be expressed as follows:

$$
\left\{\begin{array}{l}
B_{0}=-t V+x+d_{4} \varepsilon, B_{1}=-\left(2 \varepsilon / \delta_{0}\right) \arctan \left[A \tanh B_{5} /\left(d_{1}-d_{2}\right)\right], \\
B_{2}=A^{2}+\left(d_{1}-d_{2}\right)^{2}, B_{3}=-A^{2}+\left(d_{1}-d_{2}\right)^{2} \\
B_{4}=d_{1}-d_{2}-d_{1} \delta+d_{2} \delta, B_{5}=d_{3} \varepsilon+A \delta_{0} B_{0} / 2 .
\end{array}\right.
$$

(II) If $d_{1}=d_{2}$, then we get $a_{0}(t, x), a_{1}(t, x)$ as follows by substituting $\xi(z)$ into Equation (4).

$$
\begin{gathered}
a_{0}(t, x)=\frac{C_{4}+2\left[-C_{0} V+C_{2}\left(-V+C_{1}\right)\right] d_{3}^{2} \mp d_{3} C_{5} C_{6}}{2\left(C_{2}+C_{0}\right)\left(C_{2}+C_{0}-d_{3}^{2}\right)} \\
a_{1}(t, x)= \pm \frac{2 c \varepsilon d_{3}^{2}\left(A_{1}+d_{2}\right) \exp \left[C_{3}-\frac{1}{\delta_{0}} 2 \arccos \left(\frac{ \pm d_{3}}{\sqrt{C_{2}+C_{0}}}\right)\right]}{C_{6}\left(C_{2}+C_{0}\right)^{3 / 2}}
\end{gathered}
$$

where $C_{0}, C_{1}, C_{2}, C_{3}, C_{4}, C_{5}, C_{6}$ can be expressed as follows: 


$$
\left\{\begin{array}{l}
C_{0}=\exp C_{3}, C_{1}=c\left(A_{1}+d_{2}\right) d_{3} \delta_{0}, \\
C_{2}=d_{2}^{2}+d_{3}^{2}, C_{3}=d_{3} \delta_{0}\left(A_{1}+d_{2}\right)\left(d_{4}-t V+x\right), \\
C_{4}=-C_{2} C_{0}\left(-4 V+C_{1}\right)-2 C_{2}^{2}\left(-V+C_{1}\right)+C_{0}\left(2 V+C_{1}\right), \\
C_{5}=2 c d_{3} C_{0}\left(A_{1}+d_{2}\right) \sqrt{C_{2}+C_{0}}(\delta-1), C_{6}=\sqrt{1-d_{3}^{2} /\left(C_{2}+C_{0}\right)} .
\end{array}\right.
$$

Subcase 1.2:

When $d_{2}^{2}+d_{3}^{2}-d_{1}^{2}<0, \quad A^{2}=d_{1}^{2}-d_{2}^{2}-d_{3}^{2}, \quad d_{1} \neq d_{2}$,

$$
\xi(z)=\varepsilon \exp \left[\frac{2}{\delta_{0}} \arctan \left(\frac{A \tan \left(-\frac{A}{2} \delta_{0} \varepsilon\left(z+d_{4}\right)\right)+d_{3}}{d_{2}-d_{1}}\right)\right]
$$

By substituting $\xi(z)$ into (4), we get $a_{0}(t, x), a_{1}(t, x)$ as follows,

$$
\begin{aligned}
& a_{0}(t, x)= \frac{1}{2\left(D_{0}-A^{2} \tanh D_{2}^{2}\right)}\left[2\left(A^{2}+D_{0}\right) V\right. \\
&\left.-A \operatorname{sech} D_{2}^{2}\left(2 A V+D_{1}+c\left(A^{2}+D_{0}^{2}\right) \delta_{0} \varepsilon \sinh D_{3}\right)\right], \\
& a_{1}(t, x)=-\frac{2 A^{2} c\left(d_{1}-d_{2}\right) \exp \frac{2 \arctan D_{4}}{\delta_{0}} \operatorname{sech} D_{2}^{2}}{D_{0}+A^{2} \tanh D_{2}^{2}}
\end{aligned}
$$

where $D_{0}, D_{1}, D_{2}, D_{3}, D_{4}, D_{5}$ can be expressed as follows:

$$
\left\{\begin{array}{l}
D_{0}=\left(d_{1}-d_{2}\right)^{2}, D_{1}=2 A c\left(d_{1}-d_{2}\right)(\delta-1) \varepsilon \\
D_{2}=d_{3}-\frac{1}{2} A D_{5} \delta_{0} \varepsilon, D_{3}=2 d_{3}-A D_{5} \delta_{0} \varepsilon \\
D_{4}=A \tan D_{2} /\left(d_{1}-d_{2}\right), D_{5}=d_{4}-t V+x
\end{array}\right.
$$

Case 2: If $s=1$, we only give one kinds of exact travelling wave solution as follows. When $4 d_{1} d_{3}-d_{2}^{2}<0, d_{3} \neq 0, A^{2}=d_{2}^{2}-4 d_{1} d_{3}$,

$$
\xi(z)=\varepsilon \exp \left[-\frac{\varepsilon A \tanh \left(\frac{1}{2} A\left(z+d_{4}\right)\right)+d_{2}}{2 d_{3}}\right]
$$

By substituting $\xi(z)$ into (4), we get $a_{0}(t, x), a_{1}(t, x)$ as follows,

$$
\begin{gathered}
a_{0}(t, x)=\frac{4 d_{3} V+A \operatorname{csech}\left(\frac{1}{2} A E_{0}\right)^{2}\left[E_{2}+2 d_{3} \sinh \left(A E_{0}\right)\right]}{4 d_{3}} \\
a_{1}(t, x)=\frac{A^{2} c \exp E_{3}}{d_{3}\left[1+\cosh \left(A E_{0}\right)\right]}
\end{gathered}
$$

where $E_{0}, E_{1}, E_{2}, E_{3}$ can be expressed as follows:

$$
\left\{\begin{array}{l}
E_{0}=d_{4}-t V+x, E_{1}=A E_{0} / 2, \\
E_{2}=A(-1+\delta) \varepsilon, E_{3}=\left(d_{2}+A \varepsilon \tanh E_{1}\right) / 2 d_{3} .
\end{array}\right.
$$

In all of above cases, by substituting $a_{0}(t, x), a_{1}(t, x)$ and the general solu- 
tions of ODE (3) into the solution expression (6) respectively, we can obtain the exact traveling wave solutions of Equation (1).

The characteristics of these solutions are multi-layer composite solutions, i.e., they are constructed by compounding several elementary functions. For example, when $s>1$, the solutions are composed of five elementary functions exp, arctan, $\tanh , \tan , \arccos$ etc. These solutions cannot be obtained by other methods [5] [6] [7] [8]. While $s<1$ or $s=1$, the solutions will included rational or irrational solutions and soliton solutions if we select the appropriate parameters. This makes it possible for system of exact solutions for Equation (1) becomes more rich.

\subsection{The Exact Non-Traveling Wave Solutions}

In fact, Equation (5) can be written as

$$
\begin{aligned}
& \xi^{3}\left[P\left(P+2 c \xi_{x x}\right) \xi_{x x}-2 P_{x}\left(P+c \xi_{x x}\right) \xi_{x}+\left(P_{t}-a P_{x}+c P_{x x}\right) \xi_{x}^{2}\right] \\
& -c^{2} s \xi_{x}^{4}\left(\xi_{x}^{2}-\xi \xi_{x x}\right)=0
\end{aligned}
$$

where $P=\xi_{t}+c \xi_{x x}-a \xi_{x}$ for arbitrary constant $a$. We consider the following two cases for getting most general form exact solutions.

Case I. For $\xi \xi_{x x}-\xi_{x}^{2}=0$ and $s=2 \delta-\delta^{2}+4 v$.

In this case, we got $\xi(t, x)=d_{4} \exp \left[\left(d_{2} x+d_{3}\right) /\left(t+d_{1}\right)\right]$, which results in the exact solutions of Equation (1) given by (6) with (4) as follows

$$
u(t, x)=\frac{c d_{2}^{2}(\delta-1)+d_{3}+d_{2} x+2 c d_{2}^{2} \xi(t, x) \phi_{i}(\xi)}{d_{2}\left(d_{1}+t\right)}
$$

where $d_{i}$ are arbitrary constants, $\phi_{1}(\xi), \phi_{2}(\xi)$ and $\phi_{3}(\xi)$ are given in (7)-(9).

Case II. For $s=2 \delta-\delta^{2}+4 v=0$.

In this case, Equation (5) or (27) allows the solutions $\xi(t, x)$ of the linear heat equation

$$
\xi_{t}=a \xi_{x}-c \xi_{x x}
$$

with a conductive term $a \xi_{x}$ for arbitrary constant $a$.

It is fascinating that a transformation in the form of (6) between Equation (1) and (29) has been found. We will obtain infinite number of exact solutions of Equation (1) by this transformation. In addition, this connection offers us the integrability of Equation (1). For instance, we can make an investigation of the rational solutions, multi-soliton solutions and other forms of solutions to the Burgers equation by using this transformation.

In the Case II, according to the solution expression (6) with $i=2$, substituting any solution of Equation (29) into (4), we get the exact solutions of Equation (1). For example, corresponding to the solutions of Equation (29), we obtain four exact solutions $\left(u_{j}, v_{j}\right)(j=1,2,3,4)$ of Equation (1) as follows.

$$
\begin{aligned}
\text { 1. } \xi_{1}(t, x)= & A \exp \left[(x+a t)^{2} / 4 c t\right] / \sqrt{t}+B,(t, x) \in R^{2}: \\
u_{1}(t, x)= & {\left[A h_{1}(t, x)[a t(\delta-2)+x \delta]-2 a B t^{3 / 2}\right] / 2\left[A t h_{1}(t, x)+B t^{3 / 2}\right] } \\
& +A(a t+x) h_{1}(t, x) \phi_{2}\left(\xi_{1}\right) / t^{3 / 2}
\end{aligned}
$$


2. $\xi_{2}(t, x)=A \exp \left(c k^{2} t\right) \cos [k(x+a t)+B]+C,(t, x) \in R^{2}$ :

$$
\begin{aligned}
u_{2}(t, x)= & -\frac{a C+a A h_{2}(t, x) \cos \eta_{1}+A c k \delta h_{2}(t, x) \sin \eta_{1}}{2 h_{2}(t, x) \cos \eta_{1}+C} \\
& -2 c k A h_{2}(t, x) \sin \eta_{1} \phi_{2}\left(\xi_{2}\right) .
\end{aligned}
$$

3. $\xi_{3}(t, x)=A \exp [-k(x+a t)] \cos [k x+k(a+2 c k) t+B]+C,(t, x) \in R^{2}$ :

$$
\begin{aligned}
u_{3}(t, x)= & -\frac{a C+A\left[(a+c k \delta) \cos \eta_{2}+c k \delta \sin \eta_{2}\right] h_{3}(t, x)}{C+A h_{3}(t, x) \cos \eta_{2}} \\
& -2 c k A h_{3}(t, x) h_{4}(t, x) \phi_{2}\left(\xi_{3}\right) .
\end{aligned}
$$

4. $\xi_{4}(t, x)=c_{0} \operatorname{erfc}\left(\frac{x+a t}{2 \sqrt{-c t}}\right), t>0, x+a t>0$ :

$$
u_{4}(t, x)=-a+\frac{\delta \sqrt{-c t}}{\sqrt{\pi} t h_{1}(t, x) \operatorname{erfc}\left(\eta_{3}\right)}-\frac{2 c c_{0}}{\sqrt{-c \pi t} h_{1}(t, x)} \phi_{2}\left(\xi_{4}\right) .
$$

In all of above cases, $\phi_{2}(\xi)$ which is given in (8), and $A, B, C, C_{0}$ are costants,

$$
\begin{gathered}
\left\{\begin{array}{l}
\eta_{1}=B+k(x+a t), \\
\eta_{2}=B+k x+k t(a+2 k c), \\
\eta_{3}=(x+a t) /(2 \sqrt{-c t}) .
\end{array}\right. \\
\left\{\begin{array}{l}
h_{1}(t, x)=\exp \left[(x+a t)^{2} / 4 c t\right], h_{2}(t, x)=\exp \left(c k^{2} t\right), \\
h_{3}(t, x)=\exp [-k(a t+x)], \quad h_{4}(t, x)=\sin \eta_{2}+\cos \eta_{2} .
\end{array}\right.
\end{gathered}
$$

By the same manner, from the rest two solutions of Equation (29) which are given in literature [20], we should obtain additional exact solutions in different styles to the Burgers equation. We have also obtained the multi-soliton solutions and the rational solutions of the Burgers equation by using the method. Due to the lack of space, we omit the reasoning and the solution expressions.

\section{Conclusions}

In this paper, we obtained abundant exact solutions of the Burgers equation by using the generalized simplest equation method. We found a Bäcklund transformation between the Burgers equation and the constraint equation in the process of calculation. By dealing with the constraint equation, we obtained the exact solutions of Burgers equation. These solutions include the traveling wave solutions and non-traveling wave solutions. The obtained results illustrate that our method is effective and it is meaningful for further research.

When $s=0$, we obtained a connection between the constraint Equation (5) and the linear heat equation; therefore more exact solutions of the Burgers equation are constructed. But we found new constraint equation which is different from Equation (5) to other NLEEs in the study of this method. Can we construct new exact solutions to NLEEs because of these new constraint equations? It is an excellent topic for further research. 


\section{Acknowledgements}

This work is supported by National Natural Science Foundation of China (11661060, 11561051), Natural Science Foundation of Inner Mongolia Autonomous Region (2014MS0114), High Education Science Research Program of Inner Mongolia (NJZZ14053).

\section{References}

[1] Ablowitz, M.J. and Clarkson, P.A. (1991) Solitons, Nonlinear Evolution Equations and Inverse Scattering. Cambridge University Press, Cambridge. https://doi.org/10.1017/CBO9780511623998

[2] Hirota, R. (2004) The Direct Method in Soliton Theory. Cambridge University Press, Cambridge. https://doi.org/10.1017/CBO9780511543043

[3] Weiss, J. (1984) Bäcklund Transformation and Linearizations of the Henon-Heiles System. Physics Letters A, 102, 329-331. https://doi.org/10.1016/0375-9601(84)90289-5

[4] Wang, M.L., Zhou, Y.B. and Li, Z.B. (1996) Applications of a Homogeneous Balance Method to Exact Solutions of Nonlinear Equations in Mathematical Physics. Physics Letters A, 216, 67-75. https://doi.org/10.1016/0375-9601(96)00283-6

[5] Fan, E.G. and Hon, Y.C. (2003) Applications of Extended Tanh Method to Special Types of Nonlinear Equations. Applied Mathematics and Computation, 141, $351-$ 358. https://doi.org/10.1016/S0096-3003(02)00260-6

[6] Zhou, Y.B., Wang, M.L. and Wang, Y.M. (2003) Periodic Wave Solutions to a Coupled KdV Equations with Variable Coefficients. Physics Letters A, 308, 31-36. https://doi.org/10.1016/S0375-9601(02)01775-9

[7] He, J.H. and Wu, X.H. (2006) Exp-Function Method for Nonlinear Wave Equations. Chaos, Solitons \& Fractals, 34, 700-708.

https://doi.org/10.1016/j.chaos.2006.03.020

[8] Wang, M.L., Li, X.Z. and Zhang, J.L. (2008) The (G'/G)-Expansion Method and Traveling Wave Solutions of Nonlinear Evolution Equations in Mathematical Physics. Physics Letters A, 372, 417-423. https://doi.org/10.1016/j.physleta.2007.07.051

[9] Alam, Md.N., Belgacem, F.B.M. and Akbar, M.A. (2015) Analytical Treatment of the Evolutionary (1+1)-Dimensional Combined KdV-mKdV Equation via the Novel (G'/G)-Expansion Method. Journal of Applied Mathematics and Physics, 3, 1571-1579. https://doi.org/10.4236/jamp.2015.312181

[10] Kudryashov, N.A. (2005) Simplest Equation Method to Look for Exact Solutions of Nonlinear Differential Equations. Chaos, Solitons \& Fractals, 24, 1217-1231. https://doi.org/10.1016/j.chaos.2004.09.109

[11] Bilige, S.D. and Chaolu, T. (2010) An Extended Simplest Equation Method and Its Application to Several Forms of the Fifth-Order KdV Equation. Applied Mathematics and Computation, 216, 3146-3153. https://doi.org/10.1016/j.amc.2010.04.029

[12] Ali, M.Y., Hafez, M.G., Chowdury, M.K.H. and Akter, M.T. (2016) Analytical and Traveling Wave Solutions to the Fifth Order Standard Sawada-Kotera Equation via the Generalized $\operatorname{Exp}(-\Phi(\xi))$-Expansion Method. Journal of Applied Mathematics and Physics, 4, 262-271. https://doi.org/10.4236/jamp.2016.42033

[13] Yang, L. (2014) Application of Trial Equation Method for Solving the Benjamin Ono Equation. Journal of Applied Mathematics and Physics, 2, 45-49. https://doi.org/10.4236/jamp.2014.23005

[14] Lu, J.L. and Hong, X.C. (2016) Exact Traveling Wave Solutions for Generalized 
Camassa-Holm Equation by Polynomial Expansion Methods. Applied Mathematics, 7, 1599-1611. https://doi.org/10.4236/am.2016.714138

[15] Ma, W.X. and Fuchssteiner, B. (1996) Explicit and Exact Solutions to a Kolmogorov-Petrovskii-Piskunov Equation. International Journal of Non-Linear Mechanics, 31, 329-338. https://doi.org/10.1016/0020-7462(95)00064-X

[16] Ma, W.X. and Lee, J.H. (2009) A Transformed Rational Function Method and Exact Solutions to the 3+1 Dimensional Jimbo-Miwa Equation. Chaos, Solitons \& Fractals, 42, 1356-1363. https://doi.org/10.1016/j.chaos.2009.03.043

[17] Ma, W.X., Huang, T.W. and Zhang, Y. (2010) A Multiple Exp-Function Method for Nonlinear Differential Equations and Its Application. Physica Scripta, 82, Article ID: 065003. https://doi.org/10.1088/0031-8949/82/06/065003

[18] Ma, W.X. (2011) Generalized Bilinear Differential Equations. Studies in Nonlinear Sciences, 2, 140-144.

[19] Ma, W.X. (2012) A Refined Invariant Subspace Method and Applications to Evolution Equations. Science China Mathematics, 55, 1796-1778.

https://doi.org/10.1007/s11425-012-4408-9

[20] Bilige, S.D. and Wang, X.M. (2015) A Generalized Simplest Equation Method and Its Application to the Boussinesq-Burgers Equation. PLOS ONE, 10, e0126635. https://doi.org/10.1371/journal.pone.0126635

\section{Submit or recommend next manuscript to SCIRP and we will provide best service for you:}

Accepting pre-submission inquiries through Email, Facebook, LinkedIn, Twitter, etc. A wide selection of journals (inclusive of 9 subjects, more than 200 journals) Providing 24-hour high-quality service User-friendly online submission system Fair and swift peer-review system Efficient typesetting and proofreading procedure Display of the result of downloads and visits, as well as the number of cited articles Maximum dissemination of your research work

Submit your manuscript at: http://papersubmission.scirp.org/

Or contact jamp@scirp.org 